Progress in Flight Physics 5 (2013) 69-80

DOI: $10.1051 /$ eucass/201305069

(C) Owned by the authors, published by EDP Sciences, 2013

\title{
EXPERIMENTS ON RELATIVE RECEPTIVITY OF THREE-DIMENSIONAL SUPERSONIC BOUNDARY LAYER TO CONTROLLED DISTURBANCES AND ITS DEVELOPMENT
}

\author{
A. D. Kosinov, G. L. Kolosov, A. V. Panina, \\ N. V. Semionov, and Yu. G. Yermolaev \\ Khristianovich Institute of Theoretical and Applied Mechanics \\ 4/1 Institutskaya Str., Novosibirsk 630090, Russia
}

\begin{abstract}
Results of the controlled disturbance experiments in the supersonic boundary layer on a swept wing are considered. The high-frequency glow discharge technique is used for the disturbance generation. The linear development of wave trains is tested in smooth and rough surface conditions of the models. The relative receptivity of a three-dimensional (3D) boundary layer to stationary and nonstationary disturbances is estimated. Obtained that by using microroughness elements located in a swept wing boundary layer, it is possible to get a damping effect on the linear development of a spatial wave packet in the near field of the disturbances source.
\end{abstract}

\section{INTRODUCTION}

The problem of the transition origin in the supersonic boundary layer on a swept wing has been in focus for researchers more than 50 years. Such a great attention to this problem is dictated by practical needs and, besides, is due to the difficulty of the problem. Up to now, the main result for a 3D boundary layer were obtained for subsonic flows $[1,2]$. It should be stated that experiments on the linear development of controlled disturbances in a 3D supersonic boundary layer were not successfully [3] as the linearity of the pulsation development was not checked in those experiments. A 7.7 percent airfoil was used in the previous measurement so that the linear pulsation growth region was located only within the leading edge at the conditions of the experiments. Therefore, 
new stability experiments were conducted testing thin swept wings (3 percent airfoil) at low unit Reynolds numbers. It has allowed investigating the linear disturbance evolution in the supersonic boundary layer on a swept wing for natural and controlled disturbances in smooth and rough surface conditions of the models.

The paper presents new experimental data on the wave train development as well as the mean flow distortion effect for the wave train excitation and evolution in a $3 \mathrm{D}$ supersonic boundary layer.

\section{EXPERIMENTAL SETUP}

The experiments were conducted in a T-325 low noise supersonic wind tunnel of ITAM SB RAS at Mach 2 and unit Reynolds number $\operatorname{Re}_{1}=5.2 \cdot 10^{6} \mathrm{~m}^{-1}$. A wing model with the swept angle of $\chi=45^{\circ}$ was used. The swept wing model was specially designed for controlled disturbance experiments. The test surface of the model had the radius of $R=4 \mathrm{~m}$, the bottom surface was flat (3 percent profile, maximum thickness is $12 \mathrm{~mm}$ ). A source of artificial disturbances was built in the model similar to the one described in [3]. Controlled pulsations were generated by high frequency glow discharge in the chamber and they were injected into the boundary layer through an aperture of $0.4 \mathrm{~mm}$ in diameter. Disturbances in the boundary layer were measured with the help of a constant temperature hot-wire anemometer. A hot-wire probe of tungsten wire of $10 \mu \mathrm{m}$ in diameter and $1.6 \mathrm{~mm}$ in length was used.

The pulsation measurements were synchronized with the disturbance source and the sine wave generator by a special device in CAMAC (Computer Aided Measurement And Control) crate. Alternating (AC) and direct (DC) current signals from constant temperature anemometer (CTA) were written to a personal computer by using of 12-bit ADC (analog-to-digital converter) with the sampling rate of $750 \mathrm{kHz}$ and by a DC voltmeter, respectively. Four time traces of 65,536 points in length were measured and recorded in a file in each space position of the hot wire. Mean and pulsation characteristics of the flow were obtained after data processing using a standard technique $[4,5]$. The spanwise measurements were made at the fixed normal distance from the model surface and at $y / \delta \approx$ const for each $x$ position. The spanwise movement of the probe was carried out in parallel to the leading edge. In addition to this, pulsation profiles were measured by using CTA scanning system $[6,7]$. Ten overheat loadings of the hot wire were automatically switched within $2.5 \mathrm{~s}$ four times at each position of measurements [8]. It was enough to detect even a very small amplitude of controlled pulsations. Digital time traces are averaged and the Fourier transform is used in the form presented below: 


$$
\hat{A}_{f \beta}=\frac{\sqrt{2}}{T} \sum_{n=1} \sum_{j=1} A\left(t_{n}, z_{j}\right) \exp \left(i\left(\beta x_{j}-\omega t_{n}\right) \Delta t_{n} \Delta z_{j}\right.
$$

where $T$ is the length of the digital time trace; $\Delta t_{n}=t_{n-1}-t_{n} ;$ and $A\left(t_{n}, z_{j}\right)$ are the time traces of the normalized pulsation amplitude from the hot-wire anemometer.

If it was necessary, discrete Fourier transform (DFT) and the optimized length of time traces for different frequencies were used.

\section{RESULTS}

In this section, some results obtained for different surface conditions special designed swept wing will be discussed. Experimental data for a symmetrical swept wing were presented in previous EUCASS meeting and published in [9]. Comparing natural pulsation evolution in boundary layers of both wing models it was defined that disturbances have similar features, and a specially designed swept wing is possible to use for the artificial perturbation development testing. The technique that is used here in the experiments is more complicated than used before for a flat plate in controlled disturbance experiments. As the probe moving was in parallel to the leading edge, time increase that was necessary for the measurements was obtained.

\subsection{Smooth Swept Wing}

The natural pulsation development was tested in order to verify the linear region of the transition process in the boundary layer of the model. To define the region, the statistical approach was used. The typical way to see the linear disturbance development consists in verification of the normality distribution for the pulsation amplitude as it was described in [10].

Figure 1 presents the transition curve based on pulsation measurements and CTA mean voltage output that corresponds to the mean flow conditions for the probe. It was defined that there is the linear disturbance development up to the distance of $130-140 \mathrm{~mm}$ from the leading edge.

In the linear region of the perturbation development, a low-frequency pulsation growth was detected as well as amplification of disturbances at $10-30 \mathrm{kHz}$. The amplitude-frequency spectra of disturbances for different values of $x$ positions are shown in Fig. 2. Following the results, it was decided to use 10-kilohertz frequency from the sine wave generator to create the glow discharge in the chamber and to generate almost harmonic 20-kilohertz fundamental disturbance in the $3 \mathrm{D}$ boundary layer. A similar natural pulsation evolution in 3D supersonic 


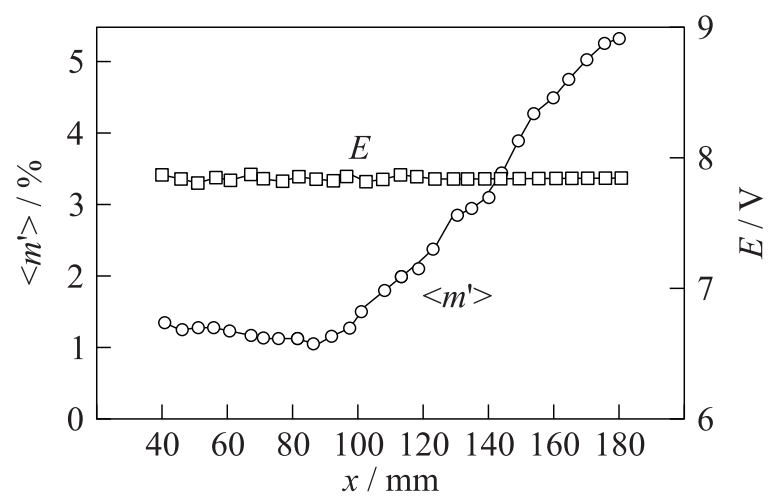

Figure 1 Total pulsation evolution data

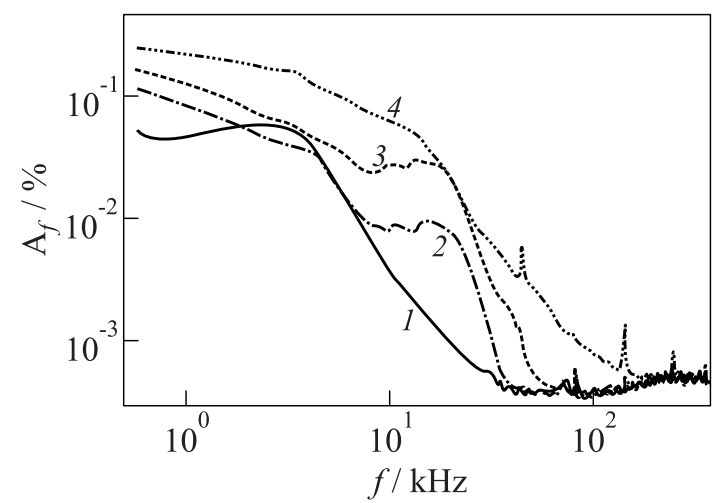

Figure 2 The amplitude-frequency spectra of disturbances for different $x$ positions: $1-\operatorname{Re}=520$ and $x=51 \mathrm{~mm} ; 2-\operatorname{Re}=767$ and $x=111 \mathrm{~mm} ; 3-\operatorname{Re}=833$ and $x=131 \mathrm{~mm} ;$ and $4-\operatorname{Re}=979$ and $x=180 \mathrm{~mm}$

boundary layer was demonstrated in [9]. From Fig. 2, it is possible to see that the maximum of the high-frequency electronic noise of the CTA is located at about $300 \mathrm{kHz}$.

Very special measurements of natural and artificial disturbance profiles were done by using scanning CTA that is similar to the device used in [6]. In general, this technique was described in [7] and was applied for the first time for flat plate measurements $[5,7]$. Then, a more powerful experiments setup was used for the supersonic boundary layer separation region testing [8]. Here, it was possible to achieve some improvement of the technique used in $[7,8]$ in order to apply it in $3 \mathrm{D}$ supersonic boundary layer measurements. 
Some comments on the dimensionless pulsation decomposition. For the supersonic flow and in the supersonic laminar boundary layer, the following condition $[11,12]$ can be considered:

$$
p^{\prime}=\rho^{\prime}+T^{\prime} \approx \text { const } .
$$

Then the density, the velocity, and the temperature fluctuations can be estimated from the following system:

$$
\left.\begin{array}{r}
\rho^{\prime}=\frac{\left(m^{\prime} \beta-T_{0}^{\prime}\right)+\alpha p^{\prime}}{\alpha+\beta} ; \\
u^{\prime}=\frac{\left(m^{\prime} \alpha+T_{0}^{\prime}\right)-\alpha p^{\prime}}{\alpha+\beta} ; \\
T^{\prime}=p^{\prime}-\rho^{\prime}
\end{array}\right\}
$$

where

$$
\alpha=\frac{1}{1+((\gamma-1) / 2) \mathrm{M}^{2}}
$$

and $\beta=(\gamma-1) \alpha \mathrm{M}^{2}$ are the well-known gasdynamic functions and $\gamma$ is the adiabatic factor.

This equation system has four unknown values that it is not enough to solve. The equations presented correspond to instantaneous variables. The mean square value of them will add some correlation terms. However, if only the linear stage of the disturbance development is considered, modules of correlation factors are equal to unity and system (1) is valid to use without additional terms after root mean squaring of the relations.

It should be mentioned that close to the upper part of the boundary layer, the following result was experimentally found out:

$$
T_{0}^{\prime}=\alpha T^{\prime}+\beta u^{\prime} \approx 0 .
$$

It is so if the sum is zero or if the temperature and velocity fluctuations are both equal to zero. The latter is correct as the eigen function of them changes its sign close to the edge of the boundary layer. These facts are enough in order to avoid isentropic relations close to the supersonic boundary layer. In this area, a special value for pressure fluctuations can be used:

$$
p^{\prime}=\rho^{\prime}=(\rho u)^{\prime}
$$

as $u^{\prime}=0$ instead of the isentropic equation [11, 12]:

$$
p^{\prime}=\gamma \rho^{\prime} \text {. }
$$

With increase of the normal coordinate, the amplitude of density fluctuation will decrease and isentropic equations will be valid for free flow:

$$
T^{\prime}=(\gamma-1) \rho^{\prime}
$$




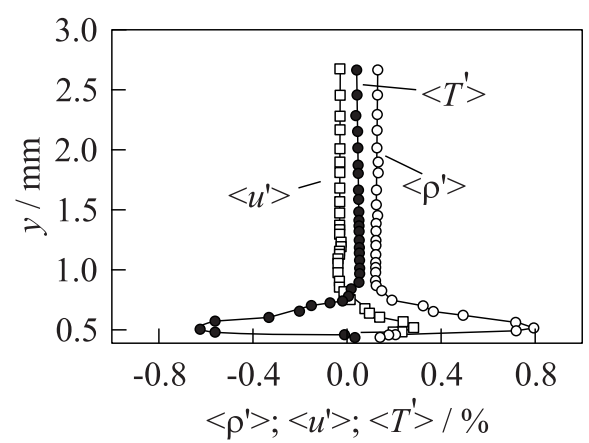

(a)

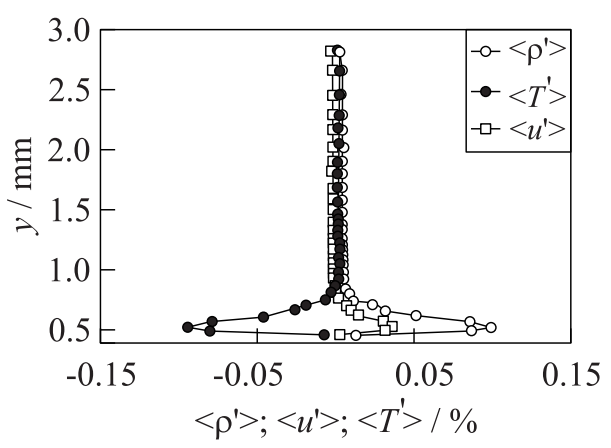

(b)

Figure 3 Pulsation profiles for natural $(a)$ and artificial $(f=20 \mathrm{kHz})(b)$ disturbances; $x=40 \mathrm{~mm}$

If pressure and total temperature pulsations are negligibly small, it is possible to obtain from (1) the Morkovin's strong Reynolds analogy:

$$
\frac{\rho^{\prime}}{u^{\prime}}=\frac{\alpha}{\beta}
$$

Let remark that far enough from the supersonic boundary layer, the isentropic relation gives at $T_{0}^{\prime} \approx 0$ for acoustic waves $[11,12]$

$$
\frac{\rho^{\prime}}{u^{\prime}}=\mathrm{M}^{2}
$$

Figure 3 shows the pulsation profiles for natural and artificial disturbances that it was possible to get with the help of such technique. Here, root mean square (rms) amplitude is presented in the manner [12] and correlation factors between pulsations $( \pm 1)$ are included. The data were measured downstream at about $97 \mathrm{~mm}$ from the leading edge or at $40 \mathrm{~mm}$ from the disturbance source in the linear region of the pulsation development. They are very similar to the results obtained before for the flat plate boundary layer, but here, there is a possibility to check from what point the isentropic relations between pulsations are valid. It will be done after additional measurements with more accuracy that is needed for controlled pulsations. Nevertheless, it is possible to expect that natural disturbance is almost isentropic out of boundary layer as it was used in $[11,12]$. For the first time, the values of velocity, temperature and density pulsations in the supersonic 3D boundary layer were estimated.

Almost harmonic wave train with the frequency of $20 \mathrm{kHz}$ was excited in the boundary layer (Fig. 4). 


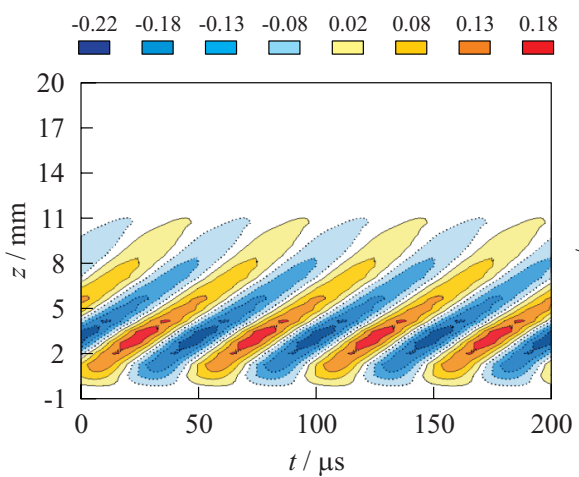

(a)

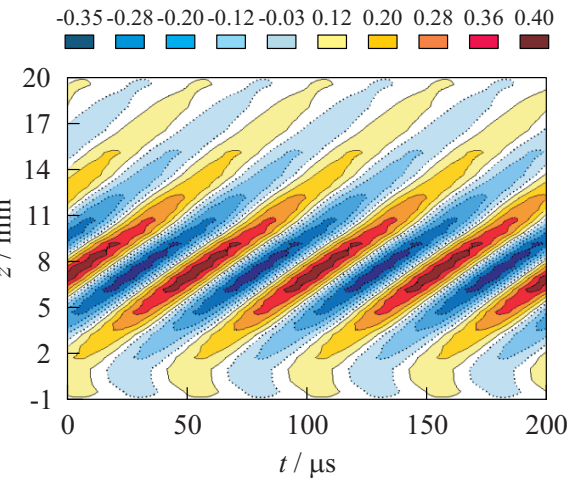

(b)

Figure 4 Time traces in spanwise direction; $X$ position is defined from the disturbance source; $f=20 \mathrm{kHz}$ : $(a) x=35 \mathrm{~mm}$; and $(b) x=75 \mathrm{~mm}$.

Similar results for periodic wave trains were detected also when 20-kilohertz disturbances were analyzed separately from another harmonics of the periodic wave train. The results indicate that a linear development for controlled pulsation in 3D boundary layer at Mach 2 was obtained.

The growth of the controlled disturbances was detected at the linear development of the natural background pulsations as it is shown in Fig. 5. Excitations of

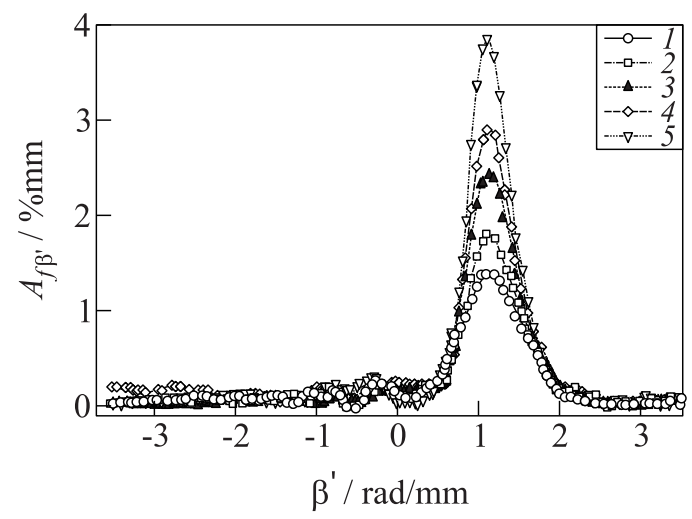

Figure 5 Wave spectra amplitude evolution for harmonic wave train; $f=20 \mathrm{kHz}$ : $1-x=35 \mathrm{~mm} ; 2-45 ; 3-55 ; 4-65$; and $5-x=75 \mathrm{~mm}$ 
other frequency disturbances were not found in the tested region. Nonlinearity was not observed. Experimentally, it was confirmed that downstream disturbances evolution on a swept wing considerably differs from the case of a flat plate. Amplitude and phase wave spectra are asymmetrical as is typical for a $3 \mathrm{D}$ boundary layer [1-3].

\subsection{On the Relative Receptivity of Three-Dimensional Boundary Layer to Roughness and Controlled Disturbances}

The other part of the paper is devoted to the study of the passive control technique of the supersonic boundary layer receptivity to controlled pulsations by means of the distributed roughness on the model surface at Mach 2. However, the first results will be concerned to the comparison of the mean flow distortion in two-dimensional (2D) and 3D boundary layers. The same single roughness is used in the flat plate and swept wing boundary layers. The experimental setup is presented in Fig. 6 . The roughness element is located at about equal boundary layer thickness conditions. Let estimate the mean flow distortion due to such a roughness. The influence of the aperture on the mean flow distortion is invisible in the near field and it was neglected. The results are presented in Fig. 7. Spanwise measurements were done at $X=76 \mathrm{~mm}$ from the leading edge for $2 \mathrm{D}$ and at $X=97 \mathrm{~mm}$ for 3D boundary layer. Here, maxima of the mean flow distortion are compared in both cases. As it follows from Fig. 7, the 3D boundary layer is more sensitive to the stationary disturbance originated from the model surface than the 2D boundary layer. The pick-to-pick stationary disturbance amplitude in the $3 \mathrm{D}$ boundary layer is about 1.5 times more than in the $2 \mathrm{D}$ boundary layer. The spanwise scale of the stationary disturbances is comparable to $Z^{\prime} \approx 1.4 Z$.

The experimental setup concerning the investigation of the mean flow distortion effect on the artificial disturbance excitation in the 3D boundary layer is presented in Fig. 8. Here, it is $X^{\prime} \approx X_{0} / 1.4$, where $X_{0}$ is measured from the
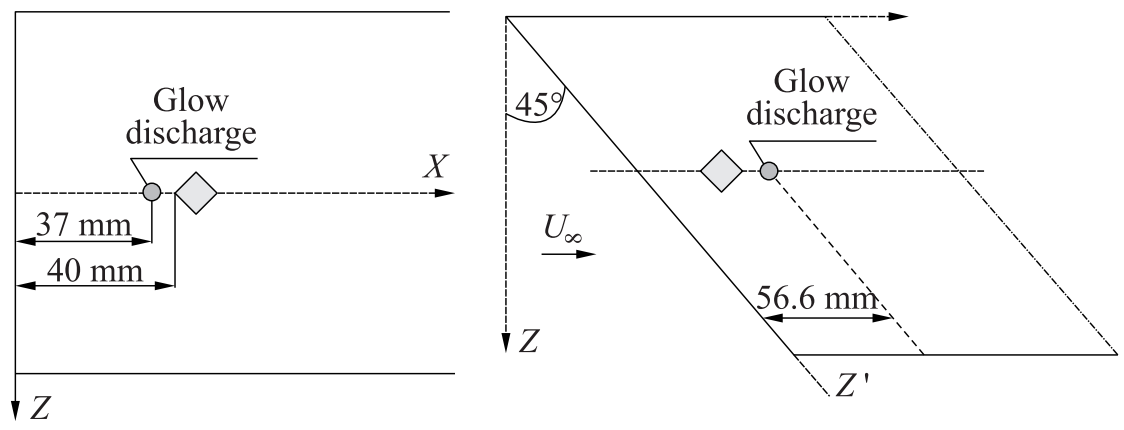

Figure 6 Experiments setup I 


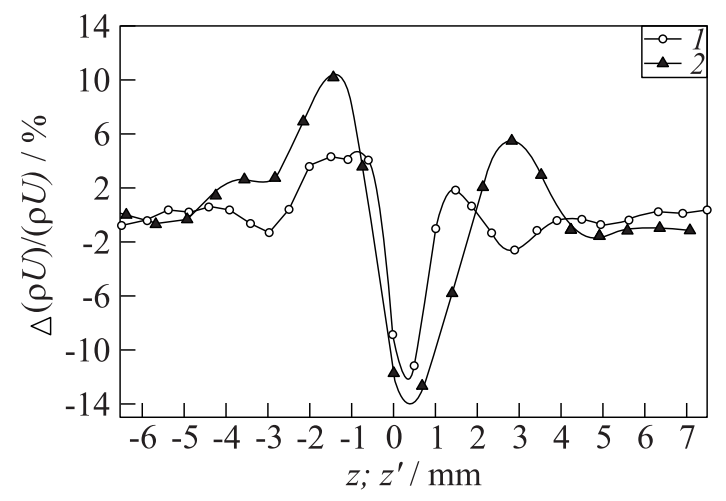

Figure 7 Mean flow distortion due to the same roughness element: 1 - flat plate; and 2 - swept wing

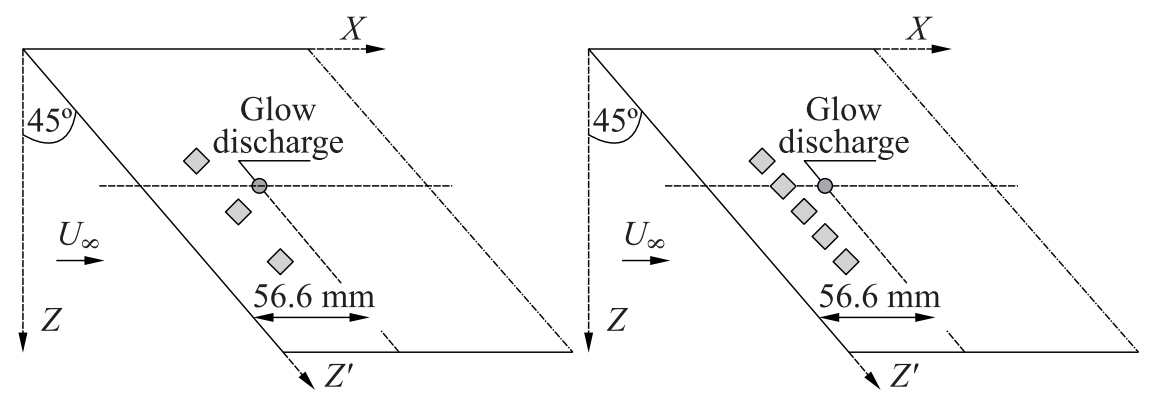

Figure 8 Experimental setup II

disturbance source. First of all, a comparison of mean flow data in spanwise direction is made for different conditions, i. e., smooth case (1), three roughness cases (2), and five roughness cases (3) that are shown in Fig. 9. For case 3, almost sine wave mean flow distortion was obtained, but when the roughness is located in one line with the source aperture, an additional distortion appears that is similar to the $2 \mathrm{D}$ boundary layer case [13].

The influence of the trip tape location and its number on the amplitude value of generated pulsations at a fixed electrical power of the glow discharge was also studied as well as at its changing. Comparative experimental data regarding the receptivity of the supersonic swept wing boundary layer to controlled disturbances for smooth and rough surfaces were obtained. Here, initial wave spectra for different surface conditions will be considered. These data are shown in Fig. 10a. As follows from this figure, the initial amplitude depends on surface roughness conditions. The maximal initial amplitude is observed in case 2 when 


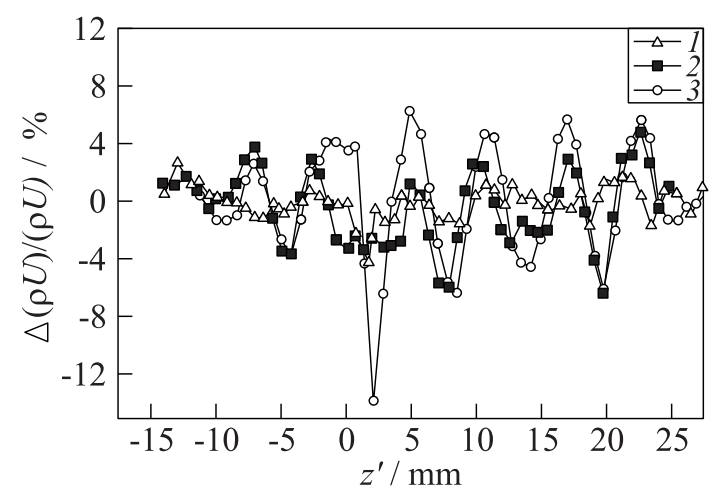

Figure 9 Mean flow distortion due to the different roughness conditions $\left(x^{\prime}\right.$ $=14.14 \mathrm{~mm}): 1$ - smooth case; 2 - three roughness cases; and 3 - five roughness cases

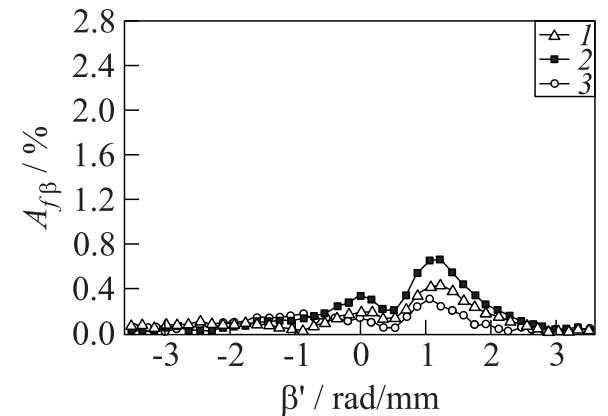

(a)

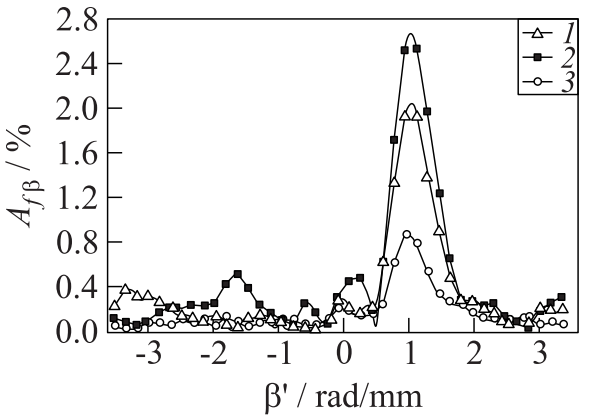

(b)

Figure 10 Comparison of wave spectra amplitude from initial $\left(x^{\prime}=14.14 \mathrm{~mm}\right)(a)$ and downstream $\left(x^{\prime}=35.36 \mathrm{~mm}\right)(b)$ spanwise distributions; $f=20 \mathrm{kHz}: 1$ - smooth case; 2 - three roughness cases; and 3 - five roughness cases

three roughness elements are located on the model surface. The minimal initial amplitude is detected in case 3 when five roughness elements are located on the model surface, and the smooth surface case 1 is between them. However, the initial amplitude spectra are quite similar to each other. The amplitude spectra shape is dramatically changed downstream due to the spanwise modulation of the mean flow only in case 2 (see Fig. 10b). Let remark that wave train amplitude increases in case 3 but relatively less than in cases 1 and 2 .

In order to see this behavior in detail, the ratio of the wave spectra amplitudes is examined. The data are presented in Fig. 11. It was obtained that damping effect of the spatial wave packet by using microroughness elements lo- 


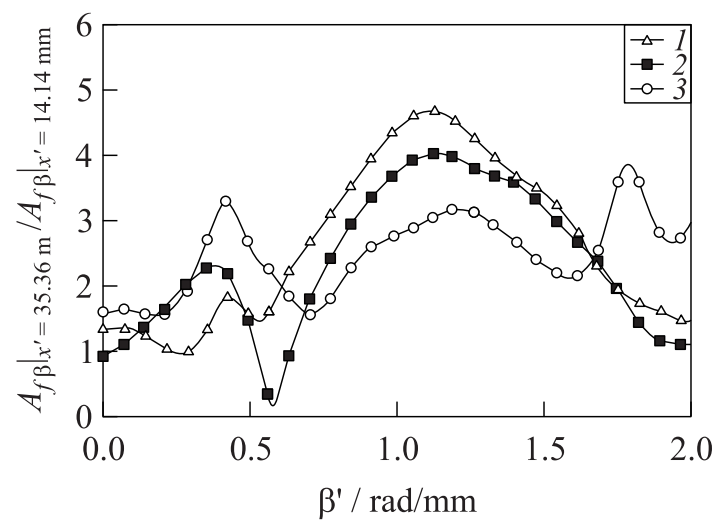

Figure 11 Relative wave spectra distributions normalised by initial wave spectra amplitude; $f=20 \mathrm{kHz}: 1$ - smooth case; 2 - three roughness cases; and 3 - five roughness cases

cated in the swept wing boundary layer can appear at least in the near field of the disturbances source. The effect is visible for most amplified waves. This effect is developed less than it was for flat plate stability experiments in [14]. The estimations are preliminary and rough but enough to allow to get the effect.

\section{CONCLUDING REMARKS}

The experimental investigation of the wave train excitation and development in the spanwise modulated flow of the supersonic swept wing boundary layer were conducted in comparison with a smooth wing surface. The results obtained give a direction for further investigations. Some of them are interesting and are worth discussing. The linear development of wave trains is tested in smooth and rough surface conditions of the models. The relative receptivity of the $3 \mathrm{D}$ boundary layer to stationary and nonstationary disturbances is estimated. It is found that the $3 \mathrm{D}$ boundary layer is more sensitive to the stationary disturbance from the model surface than the $2 \mathrm{D}$ boundary layer. It is obtained that by using microroughness elements located in the swept wing boundary layer, it is possible to get a damping effect at the linear development of the spatial wave packet at least in the near field of disturbances source.

\section{ACKNOWLEDGMENTS}

This work has been supported by the RFBR grant 09-01-00767. 


\section{REFERENCES}

1. Gaponenko, V.R., A. V. Ivanov, Y.S. Kachanov, and J. D. Crouch. 2002. Sweptwing boundary-layer receptivity to surface non-uniformities. JFM 461:93-126.

2. Saric, W. S., H. L. Reed, and E. B. White. 2003. Stability and transition of threedimensional boundary layers. Ann. Rev. Fluid Mech. 35:413-40.

3. Semionov, N. V., Yu. G. Ermolaev, A. D. Kosinov, and V. Ya. Levchenko. 2003. Experimental investigation of development of disturbances in a supersonic boundary layer on a swept wing. Thermophys. Aeromech. 10(3):347-58.

4. Kosinov, A. D., N. V. Semionov, and Yu. G. Yermolaev. 1999. Disturbances in test section of T-325 supersonic wind tunnel ITAM SB RAS. Preprint of ITAM SB RAS No. 6-99. Novosibirsk. 24 p.

5. Kosinov, A.D., Yu. G. Ermolaev, N. N. Nikolaev, N. V. Semionov, and A. I. Semisynov. 2007. On the measurements of the pulsation in supersonic boundary layer by constant temperature hot-wire anemometer. Conference (International) on the Methods of Aerophysical Research Proceedings. Novosibirsk. 5:8186.

6. Weiss, J., H. Knauss, S. Wagner, and A. D. Kosinov. 2001. Constant-temperature hot-wire measurements in a short duration supersonic wind tunnel. The Aeronautical J. R. Aeronautical Soc. 105:435-41.

7. Kosinov, A.D., A.I. Semisinov, A. Schwarz, H. Knauss, and S. Wagner. 2004. Scanning system usage of constant temperature anemometer in transition research of supersonic boundary layer. Preprint of ITAM SB RAS No. 1-2004. Novosibirsk. 20 p. [In Russian.]

8. Lenz, B., U. Gaisbauer, A.D. Kosinov, N. V. Semionov, and Yu. G. Yermolaev. 2008. Experimental investigation of the spatial propagation of artificial disturbances in the separation region of a laminar boundary layer. 14th Conference (International) on the Methods of Aerophysical Research Proceedings. Novosibirsk, Russia. I:180-81.

9. Yermolaev, Yu. G., A.D. Kosinov, and N. V. Semionov. 2011. Experimental study of stability of supersonic boundary layer on a swept wing at $\mathrm{M}=2$. TsAGI Sci. J. 42(1):3-11.

10. Semisynov, A. I., and A. D. Kosinov. 2003. On character of natural disturbances development in supersonic boundary layer on a flat plate. $J$. Thermophys. Aeromech. 10(1):41-46. [In Russian.]

11. Kovasznay, L.S. 1953. Turbulence in supersonic flow. J. Aeronautical Sci. 20:65774 .

12. Laufer, J., and T. Vrebalovich. 1960. Stability and transition of a supersonic laminar boundary layer on an insulated flat plate. J. Fluid Mech. 9:257-99.

13. Kosinov, A. D., N. V. Semionov, and Yu. G. Yermolaev. 2009. Experiments on artificial disturbances development in spanwise modulated supersonic boundary layer at Mach 2. 3rd European Conference for Aerospace Sciences (EUCASS) Proceedings.

14. Panina, A. V., A.D. Kosinov, Yu. G. Ermolaev, and N. V. Semionov. 2010. Investigation of influence of spanwise flow nonuniformity on controlled disturbances evolution in supersonic boundary layer. Vestnik NSU. Physics. 5(2):17-27. [In Russian.] 